\title{
Lente intra-ocular opaca em diplopia intratável: relato de caso
}

\author{
Opaque intraocularlens in intractable diplopia:case report
}

\author{
Fernanda Teixeira Krieger ${ }^{1}$ \\ Anelise Caron Lambert ${ }^{2}$ \\ Tatiana Contri Fraga Alves ${ }^{3}$ \\ Michele de Fátima Arruda ${ }^{4}$
}

Trabalho realizado no Instituto de Oftalmologia Tadeu Cvintal.

Mestre e Doutor em Oftalmologia pela Faculdade de Medicina de Ribeirão Preto da Universidade de São Paulo - USP - Ribeirão Preto (SP) - Brasil. Chefe do setor de Estrabismo do Instituto de Oftalmologia Tadeu Cvintal - São Paulo (SP) - Brasil.

${ }^{2}$ Médica assistente do setor de Estrabismo do Instituto de Oftalmologia Tadeu Cvintal - São Paulo (SP) - Brasil.

${ }^{3}$ Médica assistente do setor de Estrabismo do Instituto de Oftalmologia Tadeu Cvintal - São Paulo (SP) - Brasil.

${ }^{4}$ Médica residente do setor de Estrabismo do Instituto de Oftalmologia Tadeu Cvintal - São Paulo (SP) - Brasil.

Endereço para correspondência: Fernanda Teixeira Krieger. Rua Maria Figueiredo, 283 - São Paulo (SP) CEP 04002-001

E-mail: ftskrieger@hotmail.com

Recebido para publicação em 10.08.2005

Versão revisada recebida em 06.12.2005

Aprovação em 20.01.2006

\section{RESUMO}

Diplopia intratável tem sido descrita em várias situações. Métodos convencionais como prismas e correção cirúrgica do estrabismo falham em neutralizá-la. O objetivo do trabalho é documentar o caso de uma paciente com estrabismo de longa data, cuja diplopia deteriorou-se ao longo dos anos, e não foi possível resolução com cirurgia, prisma, e oclusão com óculos e lente de contato com pupila opaca. A paciente foi então submetida à facoemulsificação com implante de lente intra-ocular opaca que atingiu o objetivo desejado.

Descritores: Diplopia; Estrabismo; Implante de lentes intra-oculares; Lentes intra-oculares; Facoemulsificação; Relatos de casos [tipo de publicação]

\section{INTRODUÇAO}

A capacidade de perceber imagem única a partir do estímulo de ambos os olhos é chamada de fusão ${ }^{(1-3)}$. Ela compreende mecanismo complexo e preciso que envolve os olhos e o sistema nervoso central.

Alguns pacientes com estrabismo adquirido, sem qualquer mecanismo sensorial de supressão, permanecem com diplopia persistente ou intratável, mesmo após a obtenção de bom alinhamento ocular. Tais casos de deficiência extrema de fusão foram chamados de horror fusionis por Bielschowsky em 1935, que atribuiu os sintomas à presença de aniseiconia ${ }^{(2)}$.

Vários autores relataram sua ocorrência e discorreram sobre o possível mecanismo envolvido. Alguns deles descreveram oito pacientes com diplopia intratável e classificaram-na em absoluta e resistente ${ }^{(4)}$. Na categoria absoluta, ela está presente em todas as posições do olhar. Este tipo se divide em horror fusionis e aqueles casos com correspondência retiniana anômala que não apresentam supressão nem fusão sensorial. Na categoria resistente, os pacientes aparentemente experimentam diplopia intratável, mas são capazes de obter uma visão única binocular confortável com uso de prismas, lentes aniseicônicas ou após cirurgias. Esta categoria se subdivide em cinco tipos: estrabismo ciclovertical, correspondência retiniana anômala com supressão potencial, deslocamento da área de supressão, horror fusionis simulado e deficiência de fusão cerebral.

Em outros trabalhos ${ }^{(5-6)}$, ela foi descrita após correção cirúrgica de estrabismo.

Outro autor ${ }^{(7)}$ relatou sua presença em ângulos pequenos de desvio e propôs métodos de eliminá-la. Ele aplicou, em dois pacientes, treinamento que enfatizava a percepção da visão única periférica, evitando o uso da fusão central. O mecanismo pelo qual o método funciona não é conhecido. Em um dos pacientes, além deste tratamento, houve melhora também com a 
aplicação das tiras de Oppenheimer. Apesar do tratamento, a diplopia não desapareceu completamente, os pacientes percebiam-na quando prestavam atenção a ela, e em determinadas mudanças no tamanho e luminosidade do objeto de fixação.

Em outro estudo, os autores ${ }^{(8)}$ associaram o quadro à constante mudança do ângulo do desvio ocular, e em outro trabalho ${ }^{(9)}$ esta situação foi relatada após tratamento ortóptico. A ocorrência de diplopia persistente em pacientes sensorialmente maduros após cirurgia de catarata, com e sem antecedentes de estrabismo na infância foi descrita por alguns autores ${ }^{(10-14)}$. Segundo esses autores, a oclusão prolongada pela catarata em um olho amblíope poderia resultar na eliminação da supressão e surgimento de diplopia, em muitos casos, intratável.

A abordagem de tal situação é variada. Neste relato, apresentamos o caso de diplopia intratável de longa data, onde a paciente foi submetida à facoemulsificação com implante de lente intra-ocular (LIO) opaca, após várias tentativas, sem sucesso, de neutralizar a diplopia por métodos convencionais.

\section{RELATO DO CASO}

Paciente do sexo feminino, 65 anos. Acompanhada no serviço desde 1965, com quadro de estrabismo desde os três anos de idade, diplopia esporádica e cefaléia desde os 12 anos de idade. Acuidade visual (AV) com melhor correção óptica de 1,0 em ambos os olhos. Refração estática de -0,50 -0,50 x $20^{\circ}$ no olho direito (OD) e de $-0,50-0,50 \times 155^{\circ}$ no olho esquerdo (OE). Posição viciosa de cabeça: inclinada e girada para a direita. Exotropia de $15^{\Delta}$ e hipertropia de $14^{\Delta}$ do OD. Submetida, no período de1965 a 1991, a nove procedimentos cirúrgicos para correção do estrabismo, sendo a primeira cirurgia em 22/07/65. Tais cirurgias envolveram: retrocesso e re-retrocesso do músculo reto superior direito, ressecção e re-ressecção do músculo reto inferior direito, tenectomia do músculo oblíquo superior esquerdo, retrocesso e re-retrocesso do músculo oblíquo inferior direito. As duas primeiras cirurgias foram realizadas sob anestesia tópica, com ajuste per-operatório. Não obtivemos o relatório cirúrgico de dois procedimentos realizados por outros profissionais, os quais não nos foram enviados mesmo após solicitação. Imediatamente após todos os procedimentos a diplopia resolvia, mas retornava após período que variava de três semanas a dois meses. Após todas as cirurgias, quando a diplopia reaparecia, prismas eram prescritos, com resolução transitória do quadro. A seguir foi tentada a neutralização com os seguintes métodos, todos sem sucesso: uso de óculos com oclusão (fita translúcida recortada e colada à lente), óculos com lente opaca e oclusão, óculos com lente de $+17,00$ dioptrias-esféricas (D), lente de contato com pupila opaca ou oclusora, lente de contato oclusora com diâmetro pupilar maior, lente de contato oclusora com diâmetro pupilar maior associada à pilocarpina tópica. A diplopia tornou-se constante, e a paciente referia estar extremamente infeliz, com limitação de atividades rotineiras como dirigir carro, e com dores na coluna cervical. Avaliação ortopédica detectou alterações ósteo-articulares decorrentes do desequilíbrio oculomotor. Manifestou então desejo de que seu olho direito fosse "eliminado pela aplicação de laser na mácula" (sic). Informada que essa hipótese não seria contemplada, não compareceu às consultas durante dois anos. Retornou após esse período com a mesma queixa e exame em relação ao sistema oculomotor. Como apresentava opacidade de cristalino, que reduziu sua AV com melhor correção óptica no OD para 0,8 , decidido pela facoemulsificação com implante de LIO opaca. Após orientações quanto às possíveis complicações, e aprovação do Comitê de Ética, foi submetida ao procedimento em 05/06/04. A técnica empregada consistiu em incisão limbar superior, de 8,0 $\mathrm{mm}$ de extensão e 2,0 mm de profundidade. Realizada iridectomia periférica, extração do cristalino através de capsulorrexis de 6,0 mm. LIO marca Morcher, Alemanha, modelo D-70374, tipo 81D, com diâmetro total de $13,5 \mathrm{~mm}$, diâmetro óptico de 7,0 mm e inclinação de $10^{\circ}$, colocada no saco capsular. Incisão limbar fechada com pontos isolados de mononylon 10-0.

No pós-operatório imediato relatava que não apresentava mais diplopia e encontrava-se satisfeita com resultado. $\mathrm{Na}$ última avaliação, com 14 meses de pós-operatório, permanecia sem queixas, com AV no OD de percepção luminosa. Posição viciosa de cabeça inalterada (Figura 1). Krimsky: hipertropia do OD de $10^{\Delta}$. LIO centralizada (Figura 2) e microscopia especular de 1.392 células $/ \mathrm{mm}^{2}$, sem variação em relação ao préoperatório. Cirurgia ortopédica para melhora nas alterações da coluna cervical será realizada em breve.

\section{DISCUSSÃO}

Nos casos de diplopia intratável, métodos convencionais, como o uso de prismas e cirurgia, não apresentam resultados satisfatórios ${ }^{(2-3,8)}$. Segundo um autor ${ }^{(2)}$, o borramento da imagem de um dos olhos seria condição razoável, que deveria deixar médico e paciente satisfeitos.

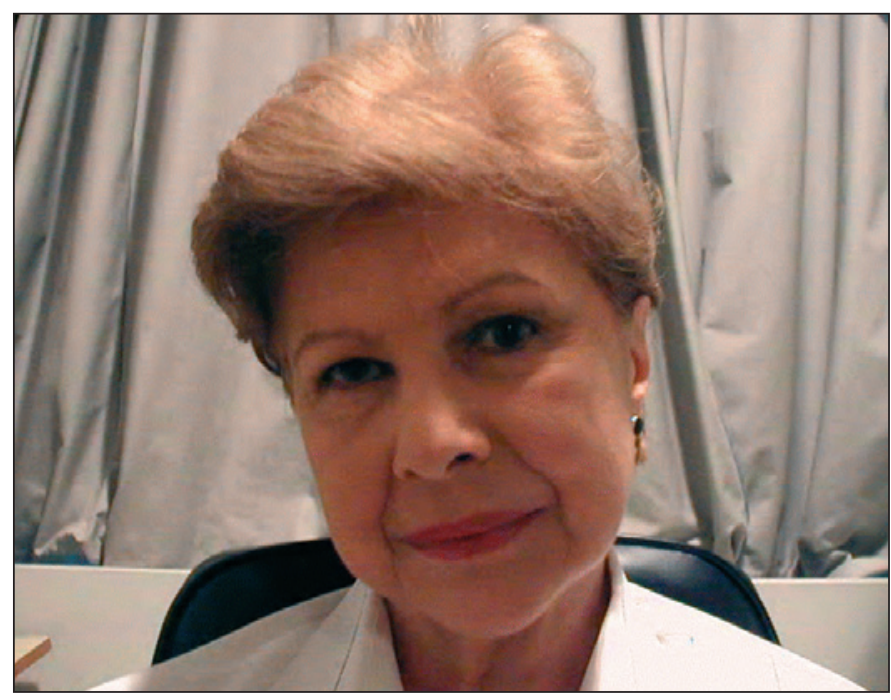

Figura 1 - Posição viciosa de cabeça: inclina e gira para a direita 


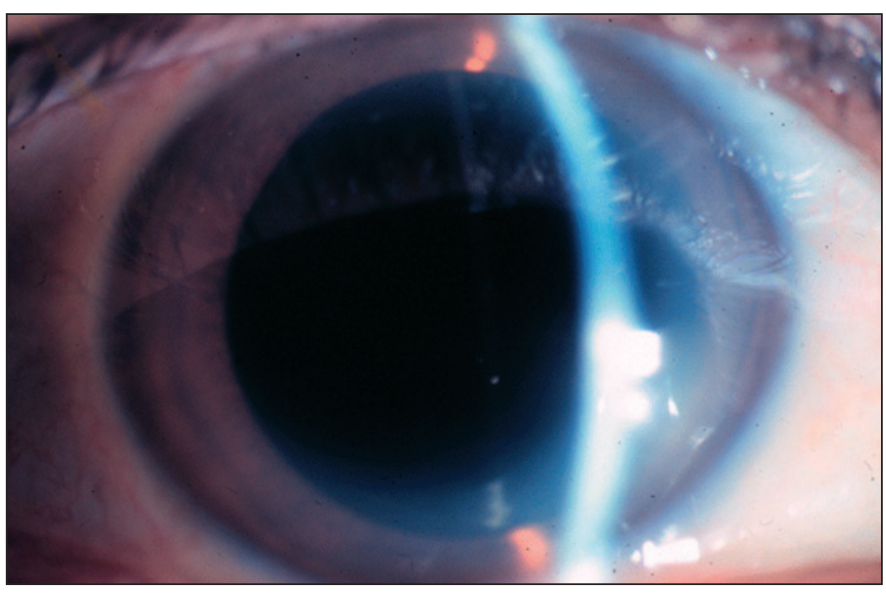

Figura 2 - Lente intra-ocular opaca em OD observada sob midríase medicamentosa

Alguns autores ${ }^{(15)}$ ressaltaram que o fracasso com tratamento padrão com prisma e cirurgia pode levar ao diagnóstico errôneo de diplopia intratável. Outros ${ }^{(16)}$ apresentaram opções de tratamento para degradar a imagem através da porção central das lentes dos óculos, de forma cosmeticamente aceitável, em casos de diplopia intratável. Isso era obtido colocando-se disco central na lente, que poderia ser translúcido, esmaltado ou possuir lente de Fresnel de +7,00 D.

Em outro estudo ${ }^{(17)}$, discorreu-se sobre o uso de lentes oclusoras em problemas de visão binocular, sendo um desses problemas a diplopia intratável. O relato de quatro pacientes tratados com lentes de contato oclusoras foi realizado por outro autor ${ }^{(18)}$, enquanto outro investigador ${ }^{(14)}$ descreveu caso onde uma lente de contato de $+20,00 \mathrm{D}$ foi adaptada para eliminar a diplopia.

$\mathrm{Na}$ paciente aqui descrita, não se conseguiu a neutralização com prismas, lentes de contato com pupila opaca e oclusores nos óculos. Antes de tais tentativas com métodos clínicos, as cirurgias de estrabismo fracassaram, apesar do fato de a paciente não apresentar diplopia, por período variável, após cada cirurgia. Como neste caso, quando não se consegue restaurar a visão binocular nem neutralizar a diplopia por meio dos tratamentos clínico e cirúrgico convencionais, o implante de LIO opaca deve ser contemplado.

Analisando a literatura pertinente, encontramos dois trabalhos onde foi colocada LIO opaca com a finalidade de neutralizar diplopia.

No primeiro deles, há o relato de um caso de diplopia intratável em homem de 25 anos com paralisia de VI nervo, submetido à correção cirúrgica do desvio, mas sem melhora do quadro. A tentativa com lente de contato oclusora também falhou em resolver o problema. Foi então implantada LIO opaca na câmara anterior, sem extração do cristalino, com sucesso ${ }^{(19)}$.

No segundo trabalho existente, os autores ${ }^{(20)}$ relataram duas pacientes com diplopia após estrabismo causado por paralisia de III e de VI nervos. Em ambos os casos não se conseguiu neutralizar a diplopia com óculos, nem lentes de contato oclusoras, quando foi então decidido pela facoemulsificação com implante de LIO opaca em câmara posterior. Entretanto, é importante ressaltar que no caso por nós apresentado a diplopia estava presente há muito tempo, devido a um desequilíbrio do sistema oculomotor desde a infância, provavelmente congênito, ao contrário dos casos até hoje descritos.

Uma piora do estrabismo poderia ocorrer após oclusão com LIO opaca, pois assim a dissociação da binocularidade seria máxima. Em 14 meses de seguimento não observamos tal complicação, tampouco existe relato na literatura nos poucos casos descritos. Porém essa situação não apresentaria dificuldade na abordagem cirúrgica, pois o procedimento para correção do estrabismo com finalidade estética oferece menor dificuldade técnica do que aquele com finalidade funcional para neutralizar a diplopia.

Como as alterações ósteo-articulares na coluna cervical são crônicas, não houve melhora após o implante da LIO opaca, nem seria esperado que isso ocorresse. Para que essa complicação pudesse ser evitada, a intervenção deveria ser muito precoce. O implante em idade tão precoce é, sem dúvida, situação de maior complexidade na indicação do procedimento. Como está agora planejado, a correção de tais alterações ortopédicas deve modificar o quadro, e desde que não há mais diplopia nem tentativa em neutralizá-la, o prognóstico de melhora é significativo.

Nos casos existentes na literatura, não há relatos de alteração endoteliais. Em um deles ${ }^{(19)}$, após seguimento de 14 anos, as alterações endoteliais apresentadas no olho com a lente de câmara anterior não foram consideradas significativas. Porém em longo prazo, com paciente jovem, como descrito por esse autor, isto é fator preocupante. Nos outros dois casos, descritos por outros autores ${ }^{(20)}$, as pacientes tinham idade de 61 e 53 anos, e o implante foi colocado na câmara posterior. Neste trabalho não há referência a possíveis alterações endoteliais. No nosso caso o implante foi colocado na câmara posterior, e a microscopia especular não se alterou no tempo de seguimento, tanto quantitativa como qualitativamente. Porém essa complicação deve ser contemplada na decisão terapêutica.

Em ambos os casos descritos por esses autores ${ }^{(20)}$ a LIO sofreu descentração, o que causava a persistência da diplopia, principalmente nas situações que favoreciam algum grau de midríase. Esta complicação foi solucionada com o uso tópico e permanente de pilocarpina a $2 \%$. O uso contínuo da pilocarpina nessas pacientes pode não ser tolerado, e nova intervenção, para um melhor posicionamento da lente, provavelmente será necessário. No nosso caso, após 14 meses de seguimento, a LIO apresenta-se bem centralizada, sem queixas de diplopia em qualquer momento do dia, nem mesmo sob midríase medicamentosa.

$\mathrm{Na}$ eventualidade de lesão grave e/ou perda de visão do outro olho e, sendo o procedimento reversível, a lente poderá ser retirada. Essa possibilidade também se aplica à presença de doenças no segmento posterior do olho com o implante. Quando se coloca LIO opaca, os pacientes devem ser orientados quanto às possíveis complicações, inerentes à facoemulsificação com implante de LIO, e também àquelas relacionadas 
com a presença da lente opaca, dificultando o diagnóstico e tratamento de doenças no segmento posterior. Nesse contexto, algumas situações contra-indicam o procedimento, como por exemplo, paciente diabético, sem necessariamente a presença de retinopatia.

Finalmente, apresentamos resultados favoráveis de um caso de facoemulsificação com implante de LIO opaca, em câmara posterior, em paciente idosa, com diplopia crônica e intratável, sugerindo que este procedimento pode ser contemplado mais precoce e freqüentemente, modificando sobremaneira a qualidade de vida desses pacientes.

\section{ABSTRACT}

Intractable diplopia has been described in many situations but poor results are the rule with standard treatment modalities. The authors report a case of a woman with long-standing strabismus and diplopia who failed to improve following surgery, prism, and occlusive spectacles or contact lenses. Then, she was submitted to phacoemulsification and opaque intraocular lens implantation, which successfully neutralized diplopia.

Keywords: Diplopia; Strabismus; Lens implantation, intraocular; Lenses, intraocular; Phacoemulsification, Case reports [publication type]

\section{REFERÊNCIAS}

1. Prietro-Diaz J, Souza-Dias C. Estrabismo. Tercera ed. La Plata: Jorge Poch; 1996. p.3-53.
2. Bielschowsky A. Congenital and acquired deficiencies of fusion. Am J Ophthalmol. 1935;18:925-37.

3. Burian HM. Fusional movements in permanent strabismus. Arch Ophthalmol. 1941;26:626-52.

4. Gruzensky WD, Palmer EA. Intractable diplopia: a clinical perspective. Graefes Arch Clin Exp Ophthalmol. 1988;226(2):187-92.

5. Tabor GL Jr. Intractable postoperative diplopia. AMA Arch Ophthalmol. 1950; 44(4):517-22.

6. Simon JW, Paskowski JR. Intractable diplopia after overcorrection of vertical strabismus. Am J Ophthalmol. 1994;117(5):675-6.

7. Birnbaum MH. Management of intractable diplopia in small angle, non-fusing squint. Am J Optom Physiol Opt. 1976;53(8):424-30.

8. Crone RA, van de Gaer L, Bonants EL. Spontaneous foveal diplopia with peripheral fusion. The coexistence of two angles of anomaly. Consequences for the theory of abnormal retinal correspondence. Arch Ophthalmol. 1970;84(2):143-7.

9. Boyd TA, Ridgway CR, Budd GE. Childhood strabismus as a cause of persistent diplopia in adolescents and adults. Can J Ophthalmol. 1966;1(3):199-205.

10. Pratt-Johnson JA, Tillson G. Intractable diplopia after vision restoration in unilateral cataract. Am J Ophthalmol. 1989;107(1):23-6.

11. Catalano RA, Nelson LB, Calhoun JH, Schatz NJ, Harley RD. Persistent strabismus presenting after cataract surgery. Ophthalmology. 1987;94(5):491-4.

12. Sharkey JA, Sellar PW. Acquired central fusion disruption following cataract extraction. J Pediatr Ophthalmol Strabismus. 1994;31(6):391-3.

13. Hamed LM, Helveston EM, Ellis FD. Persistent binocular diplopia after cataract surgery. Am J Ophthalmol. 1987;103(6):741-4.

14. Kushner BJ. Abnormal sensory findings secondary to monocular cataracts in children and strabismic adults. Am J Ophthalmol. 1986;102(3):349-52.

15. Digout LG, Awad AH. Restoration of binocular single vision after long-term fusion disruption. J AAPOS. 2003;7(3):185-9. Comment in: J AAPOS. 2005;9 (3):302; author reply 302 .

16. Kirschen D, Flom MC. Monocular central-field occlusion for intractable diplopia. Am J Optom Physiol Opt. 1977;54(5):325-31.

17. Burger DS, London R. Soft opaque lenses in binocular vision problems. J Am Optom Assoc. 1993;64(3):176-80.

18. Astin CL. The use of occluding tinted contact lenses. CLAO J. 1998;24(2): 125-7.

19. Landesz M, Worst JGF, Van Rij G. Opaque íris claw lens in a phakic eye to correct acquired diplopia. J Cataract Refract Surg. 1997;23(1):137-8.

20. Sandy CJ, Wilson S, Page AB, Frazer DG, McGinnity FG, Lee JP. Phacoemulsification and opaque intraocular lens implantation for the treatment of intratable diplopia. Ophthalmic Surgery and Lasers. 2000;31(5):429-31.

\section{0 a 22 de Outubro de 2006 Hotel Sofitel - Rio de Janeiro - RJ}

\section{INFORMAÇÕES}

$$
\begin{aligned}
& \text { Tels.: (11) 5549-2239 (Márcia) ou (21) 2553-3152 (Antonieta) } \\
& \text { Home page: www.congresso2006.retinabrasil.org }
\end{aligned}
$$

*Participação neste evento conta 10 pontos para a revalidação do título de especialista em oftalmologia 